\title{
Le réseau hub and spokes, une nouvelle donne pour les régions en marge des grands flux aériens : l'exemple de la Franche-Comté et de la Bourgogne
}

The hub and spokes network and new feature for regions on the fringe of main airlines : the example of Franche-Comté and Burgundy

"Hub and spokes » (Nabe und Speichen), eine neue Entwicklungsmöglichkeit für die Regionen am Rand des grossen Luftverkehrsströme: das Beispiel des Franche-Comté und Bourgogne (Freigrafschaft, Burgund)

\section{Madeleine Griselin et Serge Ormaux}

\section{(2) OpenEdition}

\section{Édition électronique}

URL : http://journals.openedition.org/rge/4325

DOI : $10.4000 /$ rge. 4325

ISSN : 2108-6478

Éditeur

Association des géographes de l'Est

Édition imprimée

Date de publication : 1 septembre 1999

ISSN : 0035-3213

Référence électronique

Madeleine Griselin et Serge Ormaux, "Le réseau hub and spokes, une nouvelle donne pour les régions en marge des grands flux aériens : l'exemple de la Franche-Comté et de la Bourgogne », Revue Géographique de l'Est [En ligne], vol. 39 / 4 | 1999, mis en ligne le 14 août 2013, consulté le 08 septembre 2020. URL : http://journals.openedition.org/rge/4325 ; DOI : https://doi.org/10.4000/rge 4325

Ce document a été généré automatiquement le 8 septembre 2020

Tous droits réservés 


\section{Le réseau hub and spokes, une} nouvelle donne pour les régions en marge des grands flux aériens : l'exemple de la Franche-Comté et de la Bourgogne

The hub and spokes network and new feature for regions on the fringe of main airlines : the example of Franche-Comté and Burgundy

"Hub and spokes" (Nabe und Speichen), eine neue Entwicklungsmöglichkeit für die Regionen am Rand des grossen Luftverkehrsströme : das Beispiel des Franche-Comté und Bourgogne (Freigrafschaft, Burgund)

\section{Madeleine Griselin et Serge Ormaux}

La plus grande partie des chiffres et des documents qui ont permis de rédiger cet article ont été fournis par l'Aéroport de Dijon-Bourgogne et par les compagnies aériennes y opérant. Tous nos remerciements à Messieurs Labouriaux, Foissey, et Dagot, ainsi qu'aux personnels de Regional Airlines, de Proteus Airlines, et de l'aéroport de Dijon.

1 Entre les grands aéroports qui lui sont extérieurs et le quasi vide aérien de son territoire, la Franche-Comté dispose peut-être avec l'aéroport de Dijon-Longvic (Bourgogne) d'une plate-forme aéroportuaire régionale riche de potentialités. Situé à une heure d'autoroute de la capitale comtoise, Dijon-Longvic offre en effet des vols réguliers vers un nombre assez important de villes françaises, en particulier vers l'Ouest et le Sud du pays.

2 Après avoir assuré diverses liaisons plus ou moins éphémères avec Paris, Bordeaux ou Londres, l'aéroport bourguignon a été intégré dès le début, en 1992, à la construction progressive du réseau en étoile de la compagnie Regional Airlines. 
Cette compagnie, issue de la fusion en 1992 de deux petites sociétés, Air Vendée et Air Lec, a mis en application une nouvelle conception des réseaux de transport née aux Etats-Unis : le système hub and spokes (= moyeux et rayons), appelé aussi réseau en étoile.

4 On peut se demander si la mise en place de tels dispositifs peut modifier de manière déterminante la relation des espaces concernés aux échanges aériens et leur offrir sur ce plan un véritable désenclavement. La question mérite d'autant plus d'être posée que ce type de réseau est semble-t-il en train de se développer en France et que c'est peutêtre là l'occasion de voir se recomposer l'espace aérien des régions. Afin d'apporter quelques éléments de réponse, nous avons donc cherché à savoir comment fonctionnent les réseaux en étoile auxquels Dijon est reliée et quelles possibilités réelles ils offrent aux usagers ou usagers potentiels. Au-delà du techniquement possible, nous avons ensuite évalué le degré d'intérêt de telles lignes et, pour cela, nous avons réalisé des comparaisons intermodales, en termes de rapport entre les coûts et les temps de parcours. Enfin, nous avons examiné les flux de voyageurs, c'est-à-dire la manière dont le public a répondu à l'offre nouvelle.

\section{L'intégration de Dijon à deux réseaux aériens en étoile}

$5 \quad$ Les réseaux aériens classiques, dits complexes, relient un certain nombre de villes à la manière d'une toile d'araignée; chaque aéroport est en liaison avec les autres par des vols directs, ce qui implique un très grand nombre de lignes, donc d'appareils, d'équipages et de kilomètres parcourus. En fait, ces réseaux sont rarement complets et sélectionnent les lignes les plus rentables.

6 Les réseaux en étoile sont fondés sur l'utilisation d'un aéroport-pivot (le hub ou moyeu) situé à peu près au centre de l'ensemble. Chaque ville est reliée au hub et uniquement à lui. Au lieu de la multiplicité des lignes du réseau complexe, le schéma se limite ici aux lignes en étoile (les spokes ou rayons) autour de l'aéroport-pivot. Depuis chacune des villes du réseau, on peut se rendre dans chacune des autres, en passant toujours par le hub. Une couverture aérienne très complète peut ainsi être offerte avec un nombre limité de lignes, d'avions, d'équipages et de kilomètres parcourus (Pavaux, 1995; Varlet, 1997).

7 Cette nouvelle organisation des réseaux est une conséquence de la libéralisation du transport aérien. Apparue aux États-Unis en 1978, et progressivement en Europe à partir de 1987, cette libéralisation a ouvert à la libre concurrence un marché du transport aérien qui était jusqu'alors cloisonné, protégé, soumis à des règlements étatiques et bilatéraux. Pour faire face à la nouvelle donne, les compagnies ont dû améliorer leur productivité tout en offrant à leurs passagers des services de qualité et la desserte la plus importante possible. Le hub and spokes a été l'une des réponses apportées par les compagnies au problème posé; il permet de maintenir des liaisons nombreuses, tout en faisant voler un nombre plus faible d'avions mieux remplis. Cela nécessite cependant d'organiser un système sophistiqué de correspondances afin que l'escale obligatoire dans le hub ne soit pas trop longue pour le passager, ce qui ne manquerait pas de lui faire choisir un mode de transport concurrent (voiture, train) ou une autre compagnie. 


\section{A. Le réseau hub and spokes de Regional Airlines à Clermont-Ferrand, instrument de désenclavement aérien de la Franche-Comté}

8 Le réseau couvert par Regional Airlines relie des villes et métropoles de la moitié ouest de la France à Dijon, Mulhouse-Bâle, Strasbourg, Lyon, Marseille, Toulon, Nice et, audelà, vers un arc de métropoles européennes proches : Amsterdam, Düsseldorf, Genève, Milan, Turin. Au cœur du dispositif, Clermont-Ferrand est à la fois le pivot de toutes les lignes, donc le lieu des correspondances, et le centre logistique du réseau. Quatre fois par jour, des avions venus de différents aéroports du réseau convergent sur ClermontFerrand, se rangent bord à bord, échangent leurs passagers en moins de 20 minutes et redécollent. Regional Airlines assure cinq jours par semaine deux liaisons quotidiennes aller-retour entre Clermont-Ferrand et Dijon ainsi qu'entre Clermont-Ferrand et Mulhouse-Bâle, autre aéroport proche du territoire franc-comtois; mais à partir de Dijon-Longvic, cela revient à disposer d'un vol aller-retour avec Clermont-Ferrand dans la journée et d'un aller simple en soirée, avec tout un éventail de correspondances françaises et européennes.

\section{B. La dissymétrie du réseau de Regional Airlines}

Quand on analyse en détail les destinations et les horaires aller et retour à partir de Dijon (ou de Mulhouse), il apparaît que les liaisons ne sont pourtant pas aussi fluides que le schéma théorique du hub and spokes pouvait le laisser croire.

10 Tout d'abord, le réseau est de fait partagé entre une moitié ouest et une moitié est, de part et d'autre d'un axe Rouen-Toulon. Or, depuis Dijon, donc depuis le sous-ensemble oriental, seules les liaisons avec le sous-ensemble occidental sont permises; il n'y a donc pas de possibilité, depuis Dijon, de rallier une autre ville de la moitié est telle que Amsterdam ou Nice. Cela est certes réalisable, mais sans système de correspondance et donc avec des durées bien supérieures à celles obtenues en voiture ou en train, en raison d'une rupture d'une dizaine d'heures à Clermont-Ferrand (fig.1). Vu de Besançon ou de Dijon, le réseau en étoile de Regional Airlines apparait ainsi comme une demi-étoile, on ne peut franchir la ligne de démarcation médiane que pour aller vers le secteur ouest. En d'autres termes, le réseau distingue des villes-départ et des villesarrivée et il n'est pas possible de relier deux villes-départ entre elles ou deux villesarrivée. 
Fig. 1 : Le hub aérien de Clermont-Ferrand : un réseau de deux demi-étoiles ne permettant pas de relier valablement deux villes-départ ou deux villes-arrivée entre elles (Service d'été 1998)
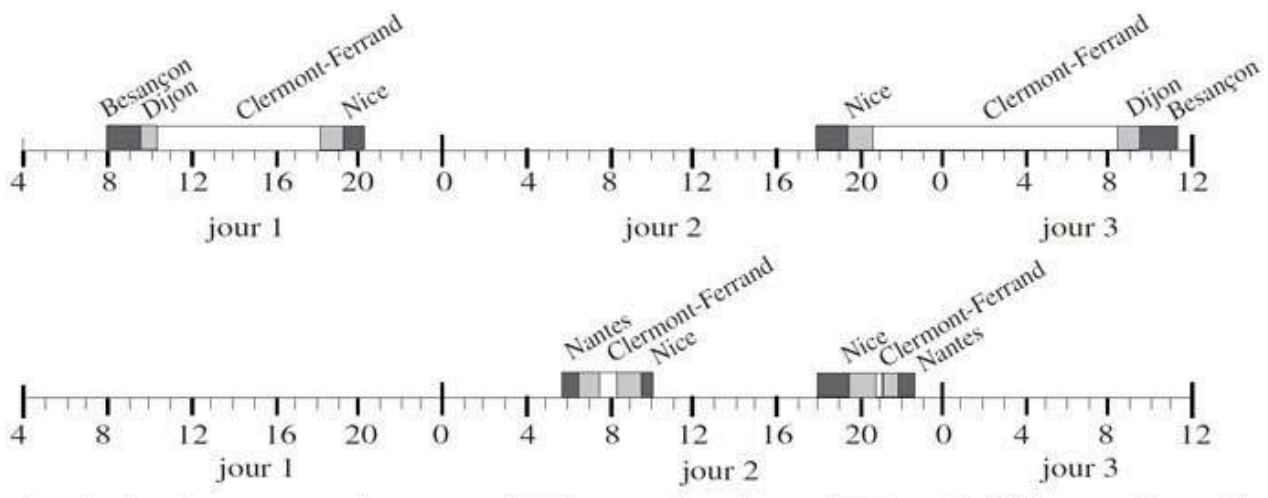

acheminement en voiture

temps de vol

$\neg$ escale à Clermont-Ferrand

Liaisons pour Nice au départ de Besançon (Dijon) et au départ de Nantes : pour un rendez-vous d'affaire d'une journée à Nice, un Nantais part durant $16 \mathrm{~h}$, un Bisontin durant 52 heures dont une nuit à Nice et une à Clermont-Ferrand ! Cela est vrai pour toutes les liaisons d'est en est du hub and spokes de Clermont-Ferrand (Dijon-Lyon, Dijon-Genève, Dijon-Milan, etc).

Mais outre cette dissymétrie spatiale, le réseau installé par la compagnie affiche également une dissymétrie temporelle, et ce au détriment des voyageurs de l'Est. Certes, le hub and spokes de Regional Airlines met une douzaine de villes de l'ouest de la France à deux heures et quart ou deux heures et demie de Dijon, donc à environ quatre heures de Besançon; cela représente un gain de temps appréciable, quand on connaît la difficulté des liaisons ferroviaires transversales, pour peu que l'on accepte la sensible différence de prix. Mais si l'on se place dans la situation, désormais classique, du professionnel souhaitant se rendre à une réunion de travail et revenir dans la même journée, l'inégalité entre l'ouest et l'est devient sensible (fig. 2).

Fig. 2 : Le hub aérien de Clermont-Ferrand : un système favorable dans le sens ouest-est (service d'été 1998)
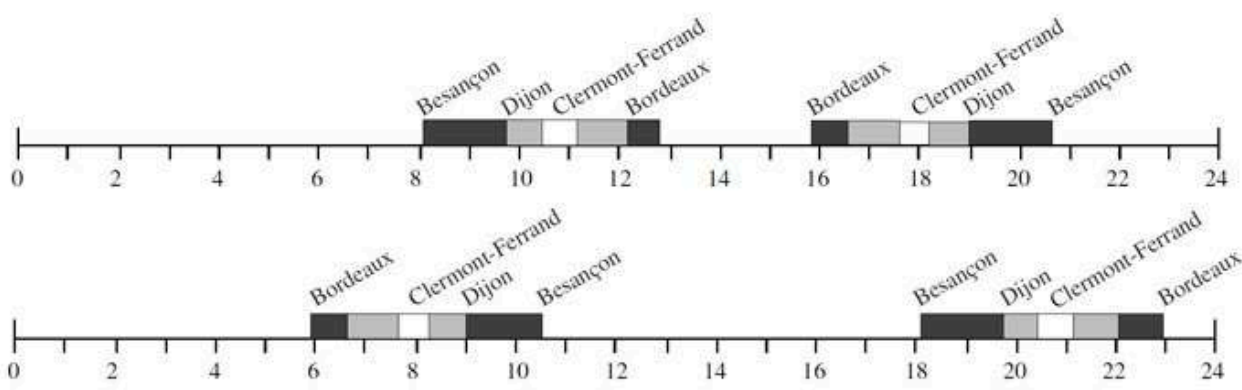

acheminement en voiture $\square$ temps de vol $\square$ escale à Clermont-Ferrand

Disponibilité sur place pour un aller-retour dans la journée entre Besançon et Bordeaux : le hub de Clermont-Ferrand est favorable dans le sens ouest-est et retour.

Un cadre d'entreprise faisant l'aller-retour Bordeaux-Dijon (et au-delà Besançon par exemple) décollera à six heures quarante-cinq de Bordeaux pour atterrir à Dijon à neuf heures moins cinq. Il disposera de toute sa journée et ne redécollera pour Bordeaux que vers dix-neuf heures quarante-cinq. Il aura passé près de onze heures sur place, de quoi se rendre sur son lieu de rendez-vous, y rester le temps nécessaire et rejoindre 
l'aéroport de Dijon. Son homologue bourguignon ou franc-comtois devra affronter un horaire beaucoup plus serré, et dans bien des cas dissuasif. Il décollera de Dijon-Longvic à neuf heures quarante-cinq, touchera le sol bordelais à midi, et devra être de retour à l'aéroport de Bordeaux-Mérignac pour le décollage à seize heures trente-cinq, ne disposant donc que de quatre heures trente pour se rendre à sa destination finale, participer à sa réunion et revenir à Mérignac!

Ces observations nous rappellent que la compagnie Regional Airlines est née dans l'Ouest de la France et a toujours son siège à Nantes; il n'est donc pas étonnant que la structure de son réseau obéisse à une logique ouest-est. En fait, les avions ne décollent pas tôt le matin de toutes les branches de l'étoile, comme on pourrait le concevoir dans un schéma théorique idéal, mais uniquement de celles de la moitié ouest. Ce sont les mêmes avions qui repartent ensuite vers l'Est pour déposer leurs passagers et embarquer enfin ceux de l'Est, ce qui repousse d'autant les décollages de Dijon, de Strasbourg ou de Mulhouse. Le soir, c'est le scénario inverse, on embarque dès le milieu de l'après-midi les Bourguignons et les Francs-Comtois à Toulouse ou Bordeaux, puis ces mêmes avions récupèrent un peu plus tard à Dijon ou Strasbourg les voyageurs venus du Sud-Ouest ; ces derniers ne redécollent ainsi qu'en début de soirée après leur longue journée passée sur place. Cela explique aussi pourquoi les gens de l'Est peuvent alors emprunter un aller simple en direction de l'Ouest ou du Sud-Ouest.

\section{La complémentarité du réseau en étoile de Proteus Airlines à Saint-Étienne}

Le 15 juillet 1997, la compagnie Protéus Airlines installait un nouveau réseau en étoile, dont le pivot est centré quant à lui à Saint-Étienne. Certaines destinations au départ de Dijon sont communes avec celles de Regional Airlines (Bordeaux, Nantes, Toulouse), d'autres sont différentes mais surtout, ce réseau est organisé depuis l'Est et, si l'on peut dire, au bénéfice de l'Est (fig. 3). En effet, les décollages de Dijon-Longvic et dans une demi-douzaine d'aéroports de la moitié Est de la France (Mulhouse n'est pas concerné pour l'instant) sont programmés vers six heures du matin, pour des arrivées qui se situent vers $8 \mathrm{~h} 30$. Les retours se font autour de 19 heures, avec atterrissage vers 21 h 25 à Dijon, Reims, Annecy, Chambéry, Avignon ou Perpignan. Dans ces conditions, le voyageur a pu disposer d'une bonne dizaine d'heures sur place, contre quatre heures trente dans la configuration de Regional Airlines. Par ailleurs, quelques liaisons Est-Est sont désormais possibles, comme Dijon-Nice ou Avignon-Strasbourg. 
Fig. 3 : Le hub Regional Airlines, de Clermont-Ferrand (A et B), et le hub Proteus Airlines, de SaintÉtienne (C et D), (été 1998)

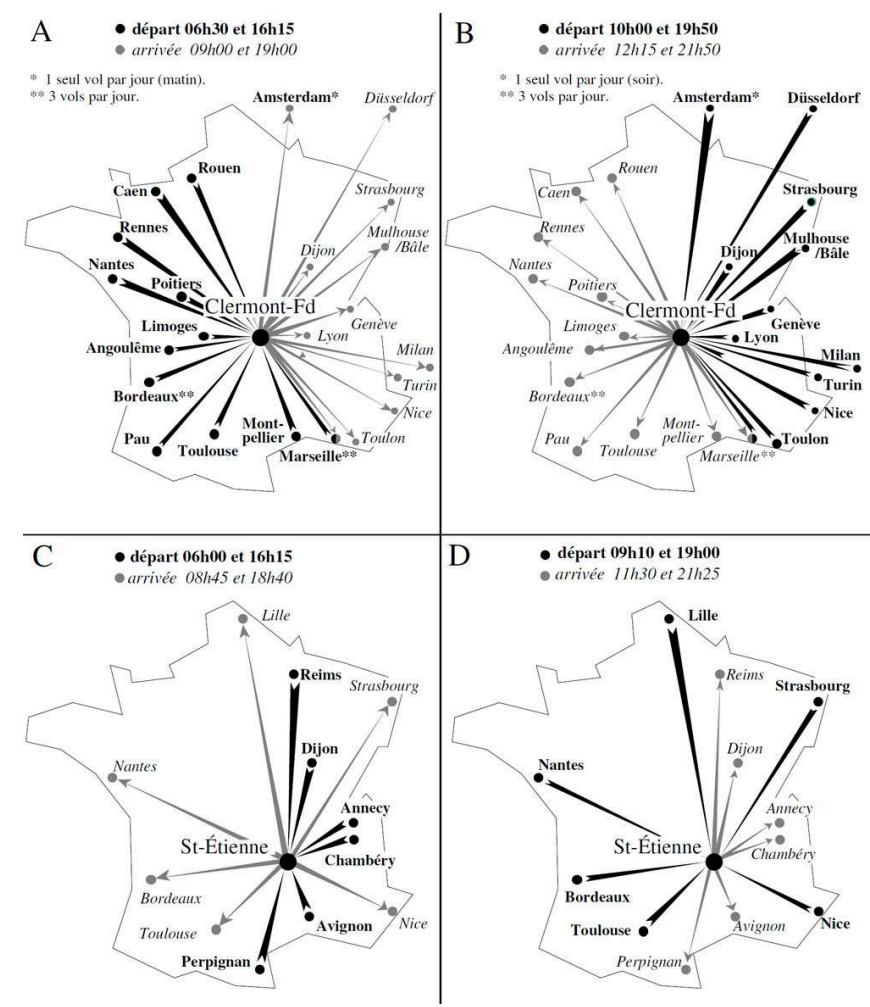

En revanche, les aéroports de l'Ouest, comme Bordeaux, Toulouse ou Nantes n'ont, avec Proteus Airlines, pas de liaisons entre eux. Quant aux voyageurs qui se déplacent de ces aéroports en direction de l'Est, ils n'ont guère la possibilité de faire avec Proteus Airlines l'aller-retour dans la journée, sauf à se contenter de quelques heures sur place. Pour eux le réseau de Regional Airlines est plus intéressant, en particulier pour les allers-retours vers l'Est dans la journée, comme nous l'avons montré plus haut.

Il y a donc une complémentarité entre les deux réseaux, accentuée encore par la proximité des deux hubs et par la similarité des appareils utilisés, des avions de dixneuf places pour les deux compagnies, au départ ou à destination de Dijon (fig. 4). 
Fig. 4 : Dijon dans les hubs de Regional Airlines et de Proteus Airlines (été 1998)

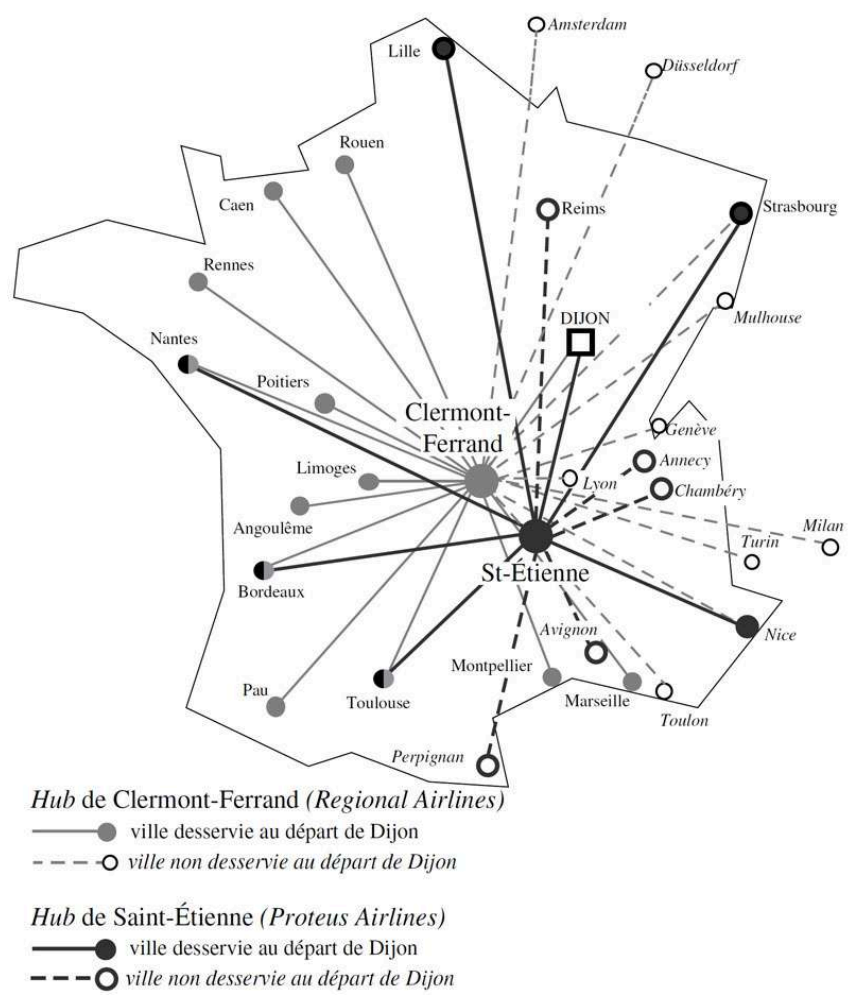

\section{La comparaison intermodale de l'offre}

Avant de voir si cette complémentarité spatio-temporelle des deux réseaux se traduit dans les flux de passagers, il est indispensable de comparer les durées et les coûts de trajets des différents modes de transport utilisables pour les liaisons concernées. Audelà de la structure des réseaux, le rapport entre le gain de temps réalisé et le surcoût occasionné est en effet un facteur essentiel dans la prise de décision des utilisateurs.

De tels comparatifs sont cependant toujours délicats à établir ; de nombreux éléments entrent en jeu, en particulier pour les coûts, ce qui implique un protocole transparent. Nous avons délibérément choisi de proposer un comparatif dont la validité s'entend pour le voyageur moyen, simple particulier, voyageur de commerce ou responsable d'une petite entreprise, qui en général ne prend pas l'avion mais utilise plutôt le train ou sa voiture et peut se demander si les nouvelles opportunités offertes par les réseaux aériens en étoile sont à sa portée sur le plan financier. Deux modes de transport existaient jusqu'alors, un troisième apparaît, comment se situe-t-il par rapport aux deux autres sur le plan du coût et de la durée du voyage? Nos calculs ont donc été réalisés à partir des valeurs standard communiquées par les serveurs Minitel destinés aux usagers.

Pour les durées des voyages en voiture, on a retenu les trajets aller simple porte-àporte les plus rapides, c'est-à-dire avec autoroutes, de 3615 ITI. Les coûts intègrent les péages autoroutiers et la distance à raison de $1,23 \mathrm{~F}$ du kilomètre, soit l'indemnité versée par l'administration à ses agents pour une voiture de cinq chevaux fiscaux (petite voiture ou voiture de catégorie moyenne avec moteur turbo-diesel, souvent utilisées par les commerciaux). 

lent et l'avion le plus rapide. Seule la liaison Dijon-Strasbourg par Saint-Étienne affiche des temps aériens supérieurs aux temps routier et ferroviaire, mais on est ici aux limites de l'utilisation du réseau car sur deux branches faisant un angle très fermé. En règle générale, le match de vitesse oppose donc l'avion et la voiture; les avantages du premier ne sont certes pas aussi importants que pourrait le laisser supposer la simple comparaison des vitesses pures pratiquées par les deux types de transport, mais sont loin d'être marginaux. Sur les 17 destinations disponibles, huit, situées dans un grand quart sud-ouest, autorisent un gain de deux heures ou plus par rapport au mode de transport terrestre le plus rapide. L'une d'entre elles, Pau, affiche même une différence de trois heures. Or, ces valeurs correspondent à des allers simples, il convient donc de doubler les gains de temps si l'on veut apprécier l'intérêt que peut représenter l'utilisation de l'avion, en particulier dans le cadre d'un déplacement bref. En valeur relative [ (temps terrestre le moins longtemps d'avion)/temps terrestre ${ }^{*} 100$ )], les résultats modèrent quelque peu l'enthousiasme, les dix destinations les plus performantes se distribuent entre un tiers et cinquante pour cent de temps gagné par rapport à la route, avec un maximum à $53 \%$ pour Pau. 
Fig. 5 : La concurrence rail-route-air, en temps et en coût, pour un usager de Besançon (juillet 1998)

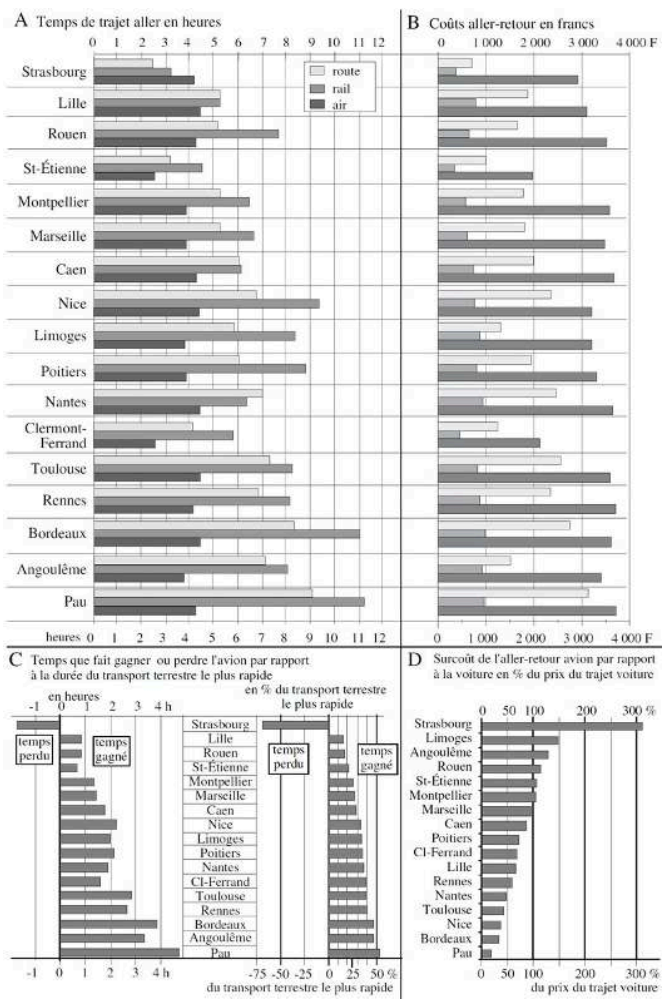

Sur le plan des coûts, l'écart est dans la plupart des cas très important entre les deux modes de transport en commun que sont l'avion et le train, mais il serait moindre si l'on avait pris comme référence le billet de première classe, sans oublier que, dans un certain nombre de cas, le voyageur aérien peut bénéficier de tarifs réduits (tarif " eurobudget " pour hommes d'affaires, billet congrès, etc.). Par rapport à la voiture, les écarts sont en général nettement plus faibles, dès lors que les coûts effectifs du déplacement ont été intégrés (ils seraient encore plus faibles si l'on avait réalisé le calcul avec des frais de déplacement par kilomètre plus généreux que ceux de l'Administration!). C'est d'ailleurs pour les destinations qui offrent le plus important gain de temps que l'écart est le plus faible : pour Bordeaux, un surcoût de $32 \%$ autorise ainsi un gain de temps de $46 \%$, le record étant établi par la liaison avec Pau, pour laquelle une rallonge de prix de $18 \%$ permet de diviser par deux le temps de transport ! Nantes, Toulouse et Rennes restent également dans des rapports surcoût/bénéfice tout à fait raisonnables.

La question de la concurrence entre l'avion et la voiture est donc d'autant plus délicate que parmi les trois modes à la disposition de l'usager, ils sont à la fois les plus rapides et les plus chers, donc les plus proches en termes de bilan. L'obtention par la voiture de la deuxième place pour le coût et la rapidité pose par ailleurs la question du nombre d'individus devant se déplacer ensemble. Si les comparatifs que nous avons proposés placent la voiture en brillant second, nous devinons sans peine qu'à partir de deux personnes transportées, le rapport surcoût/gain de temps atteint très vite des niveaux rédhibitoires, en tout cas sur le plan strictement financier.

Enfin, notre hypothèse de travail a supposé une liaison Besançon-ville aéroportuaire desservie. Si le voyageur franc-comtois se rend dans une ville non pourvue d'un aéroport relié par hub à Dijon, le gain de temps engendré par l'avion s'amenuise, et le 
coût du voyage en avion est augmenté du prix d'une jonction routière correspondant à quatre trajets pour une voiture d'accueil, ou d'une location de véhicule et d'un allerretour avec celui-ci. L'exemple du Besançon-Quimper (fig. 6) confirme ainsi le très faible coût du train, par ailleurs guère plus lent sur ce trajet que la voiture, et montre que le surcoût lié à l'avion s'accroît de manière forte avec l'éloignement entre l'aéroport de destination et le lieu de destination. L'avion facture ici d'un surcoût de $70 \%$ un gain de temps marginal de $20 \%$ !

Fig. 6 : Concurrence rail-route-air entre deux villes éloignées d'un aéroport du réseau : l'exemple de Besançon-Quimper (juillet 1998)

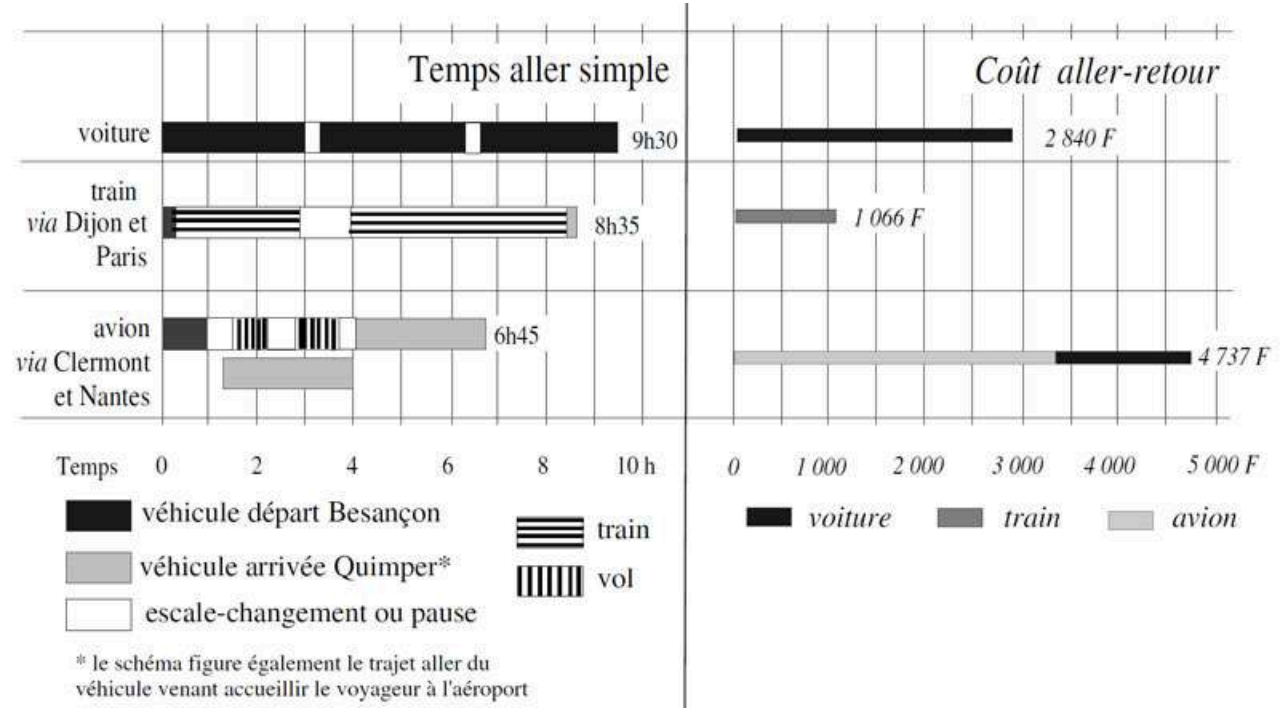

Les réseaux en étoile régionaux proposent donc une offre abondante mais avec une pénalisation de temps due à l'escale obligatoire et dans certains cas à un détour non négligeable. Ils sont donc particulièrement sensibles à tout handicap supplémentaire lié à l'éloignement des aéroports de départ et de destination. Dans le cas de Besançon, situé à une heure de route de l'accès au réseau, le recours à l'avion se justifie lorsque la destination est lointaine, plutôt diamétralement opposée, c'est-à-dire positionnée dans le Centre-Ouest ou le Sud-Ouest, mal reliée par autoroute ou par TGV et proche d'un aéroport desservi par le réseau! Plus que le décompte du temps, l'essentiel réside en fait dans l'environnement temporel du voyage. Peu rentable dans le cas d'un déplacement de plusieurs jours qui relativise le gain de temps et le fait payer cher, le recours aux réseaux en étoile offerts aux Francs-Comtois par Dijon devient intéressant lorsque le déplacement doit être court. Il peut même s'avérer particulièrement rentable sur les destinations pour lesquelles l'avion permet de substituer un aller-retour dans la journée au voyage de deux ou trois jours que nécessiterait la voiture ou le train : Il est désormais possible aujourd'hui pour un industriel ou un universitaire franc-comtois de se rendre à Bordeaux le matin, d'y disposer d'une journée complète de travail sur place et d'être de nouveau chez lui avant minuit. L'avantage comparatif sur ce style de déplacement rend d'autant plus pertinente la mise en place de la deuxième demi-étoile centrée sur Saint-Étienne. L'étude des flux va maintenant permettre d'en vérifier la validité. 


\section{L'utilisation des deux réseaux aériens ou l'analyse de la demande réalisée}

Comparé à son voisin de Dole, qui n'est concerné que par les charters et les avions taxis (Griselin \& Ormaux, 1998), l'aéroport de Dijon-Bourgogne est spécialisé dans les vols réguliers, puisque, sur les 20000 passagers l'ayant fréquenté en 1996, 62\% empruntaient les lignes régulières, $25 \%$ les charters et $13 \%$ les avions taxis.

En 1997, 12189 passagers sont arrivés à Dijon ou en sont partis à bord des avions réguliers des deux hubs.

Proteus Airlines étant entré en activité à partir de juillet 1997 à Dijon, il est intéressant de suivre l'évolution du trafic sur les six derniers mois de 1997 et les six premiers de 1998 (fig. 7).

Fig. 7 : Les flux de passagers des lignes régulières à l'aéroport de Dijon en 1997 et 1998, toutes liaisons confondues, et comparaison avec les vols charters

nombre de

passagers

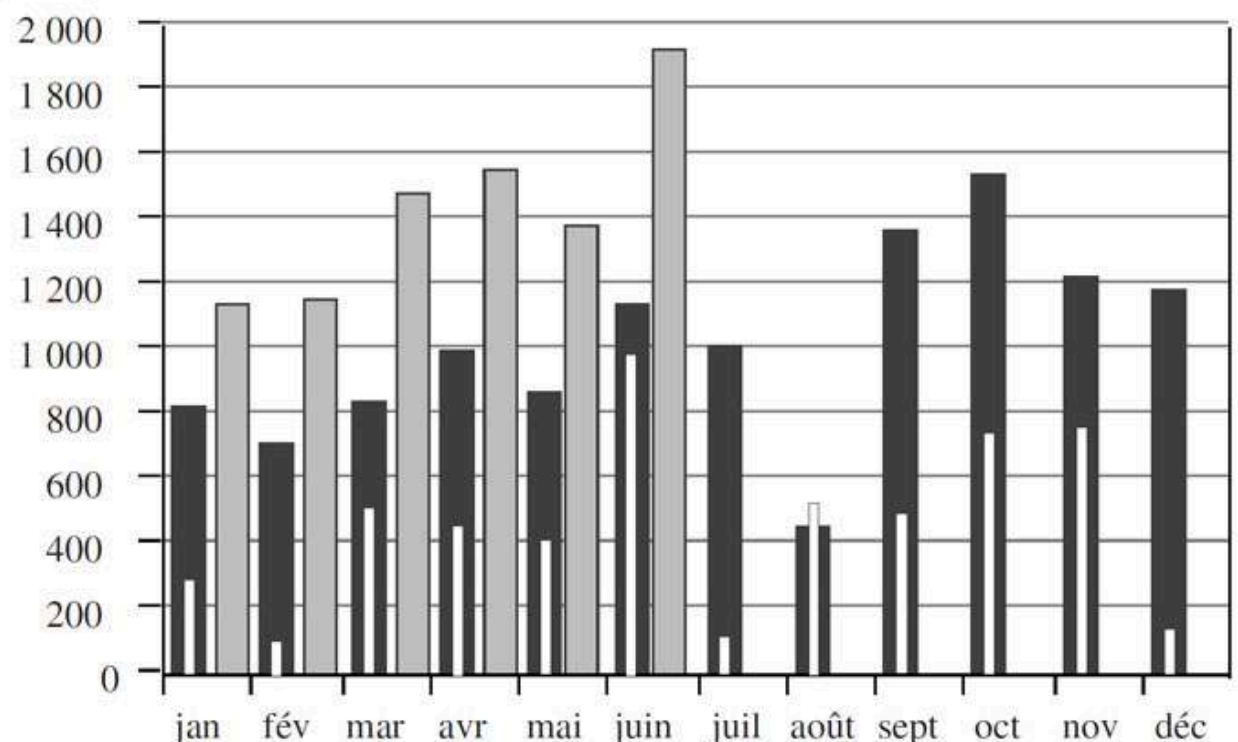

Source : Aéroport de Dijon-Bourgogne

Si le score global de 1997 (12 189 passagers réguliers) est proche de celui de 1996 (12 300), le bond en avant pourrait être significatif en 1998, puisque, sur les six premiers mois, on constate déjà une augmentation de $35 \%$ par rapport au total de passagers réguliers transportés (de janvier à juillet: 8646 en 1998 contre 6401 en 1997).

Les lignes régulières desservant Dijon ne fonctionnent que du lundi matin au vendredi soir, signe d'un service tourné davantage vers la clientèle d'affaires que vers le tourisme. Cette tendance se retrouve dans l'analyse mensuelle des fréquentations : une nette décroissance des flux en août tranche avec la reprise en septembre et octobre, mois qui affichent les plus gros trafics en 1997. 
33 À titre de comparaison, la vocation résolument touristique des vols charters décollant ou atterrissant à Dijon se lit dans un trafic plus intense en août qui est le quatrième mois en flux de passagers charter.

Il est révélateur aussi de voir que $37 \%$ des 72 vols charter ont eu lieu les samedis, dimanches et jours fériés... chiffre montant à $51 \%$ si on y inclut les vendredis.

En prenant, à titre d'exemple, le mois d'avril 1998 (mois assez significatif ne comportant qu'un jour férié), on constate que pour les vols réguliers, la répartition des passagers tout au long de la semaine est assez homogène avec cependant une nette avance du lundi qui, malgré celui de Pâques - délesté de vol régulier -, représente pour avril 1998, 27 \% du flux sur Bordeaux, le vendredi n'arrivant qu'en troisième position avec $68 \%$.

Si l'on raisonne sur les flux par destination pour une année commençant au 1er juillet 1997 et finissant au 30 juin 1998 (fig. 8), on obtient un total de 15445 passagers (entrants ou sortants) ayant voyagé sur les 18 liaisons assurées par Regional Airlines (11), par Proteus Airlines (4) ou par les deux compagnies (3) ${ }^{1}$.

Fig. 8 : Aéroport de Dijon : flux de passagers (départ et arrivée) sur les lignes de Regional Airlines (via Clermont-Ferrand) et Proteus Airlines (via Saint-Étienne) du 01.07.1997 au 30.06.1998

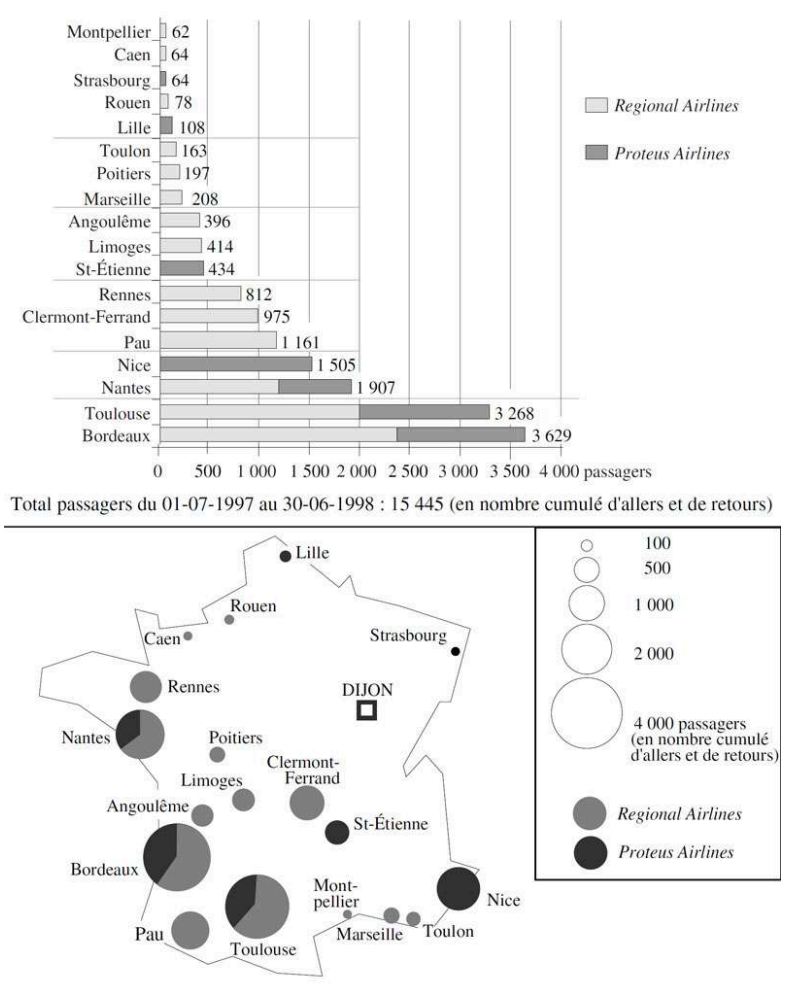

Les aéroports-pivots ne sont pris en compte dans ces deux schémas que comme destinations finales au départ de Dijon.

Source : Aéroport de Dijon-Bourgogne

Ce sont les trois destinations communes aux deux sociétés qui sont, et de loin, les plus fréquentées: Bordeaux et Toulouse dépassent les 3000 passagers annuels, Nantes approche les 2000 . Sur ces trois destinations, l'avion offre au voyageur, de villeaéroport à ville-aéroport, un gain de temps de 30 à $50 \%$ par rapport à la voiture ou au 
train ; ce sont trois destinations des moins pénalisantes, puisque le surcoût de l'avion représente $39 \%$ du prix du trajet voiture pour Bordeaux et $57 \%$ pour Nantes.

Nice, avec 1505 passagers pour 1997-1998, est la destination reine de Proteus Airlines, tandis que Pau, avec 1161 passagers, obtient la quatrième place chez Regional Airlines, talonnant Nantes sur cette compagnie (1 168). Pau offre aux voyageurs passant par Dijon le meilleur rapport gain de temps et surcoût, puisque, en prenant l'avion, il gagne 53 à $58 \%$ de temps, selon qu'il part de Dijon ou de Besançon, pour un surcoût de 16 à $18 \%$ seulement par rapport à la voiture. Comparativement, Nice est moins bien placé pour le Bisontin avec $34 \%$ de gain de temps pour un surcoût de $36 \%$.

En dessous des 1000 passagers annuels se trouve Clermont-Ferrand, liaison qu'il faut comme Saint-Étienne - considérer avec réserve : le personnel dijonnais de chacune des deux compagnies aériennes partant souvent pour son hub respectif vient gonfler les fréquentations des deux villes moyeux.

Rennes, bien que ville moins attractive en affaires que Toulouse ou Bordeaux, réalise un score confortable avec 812 passagers pour l'année, reflet du gain de temps notable (40\%) que permet l'avion pour un surcoût, tout de même pénalisant, de $59 \%$ par rapport à la voiture au départ de Besançon.

41 Angoulême (396 passagers) est dans le même cas : deuxième en gain de temps (47\%) juste derrière Pau, la liaison aérienne provoque un surcoût de $127 \%$ totalement décourageant pour un Bisontin, sauf pour un voyage d'une journée. Limoges (414 passagers) est encore plus typique, puisque le gain de temps de $35 \%$ engendre un surcoût de près de $150 \%$.

Marseille et Poitiers, aux fréquentations comparables, n'offrent pas le même avantage. Marseille, plus attractive pour les affaires, justifie le quasi doublement du prix voiture (surcoût de $94 \%$ ) pour un gain de temps inférieur à $30 \%$ depuis Besançon. La liaison avec Poitiers est légèrement mieux placée : $36 \%$ de temps gagné sur la voiture pour un surcoût de $71 \%$.

Quant aux cinq dernières destinations, leurs flux de passagers sont à analyser avec prudence car les chiffres de l'année ne font état que de 3 à 8 mois d'activité : liaison fermée fin 1997 comme Toulon (6 mois), ou ligne nouvellement ouverte comme Strasbourg (8 mois), Caen et Montpellier (3 mois).

La liaison Dijon-Lille, abandonnée par Regional Airlines en avril 1997 a été reprise par Proteus dès son installation à Dijon: les 108 passagers comptabilisés sur les 12 mois considérés reflètent le faible intérêt de la liaison qui n'apporte que $16 \%$ de gain de temps (moins d'une heure) à un automobiliste de Besançon pour un surcoût de $65 \%$, tandis que le Dijonnais perd une demi-heure en avion par rapport au TGV pour un coût équivalent à plus de trois fois l'aller-retour en TGV.

Du reste, n'est-il pas psychologiquement déroutant, pour un voyageur, de partir vers le sud pour aller vers le nord, configuration qui pénalise aussi Strasbourg, si attractive que puisse être la ville du Parlement européen; totalement inintéressant pour un Bisontin, le vol coûte plus de quatre fois le prix du trajet voiture tout en durant près d'une heure de plus. Cette destination n'est pas plus intéressante au départ de Dijon: trajet aérien plus long de 15 minutes facturé près de trois fois le prix de la voiture et six fois celui du train!

46 La configuration des deux hubs and spokes desservant Dijon confère une variabilité intermensuelle des flux significative pour chacune des cinq premières liaisons 
empruntées à Dijon. Cette variabilité traduit l'idée que les lignes régulières de l'aéroport bourguignon sont optimales pour une clientèle d'affaires ou aisée, clientèle pressée, partant à la journée, pour qui les critères de comparaisons retenus plus haut jouent peu (fig. 9).

Fig. 9 : Flux mensuel des cinq liaisons aériennes les plus fréquentées à l'aéroport de Dijon du 01.07.1997 au 30.06.1998

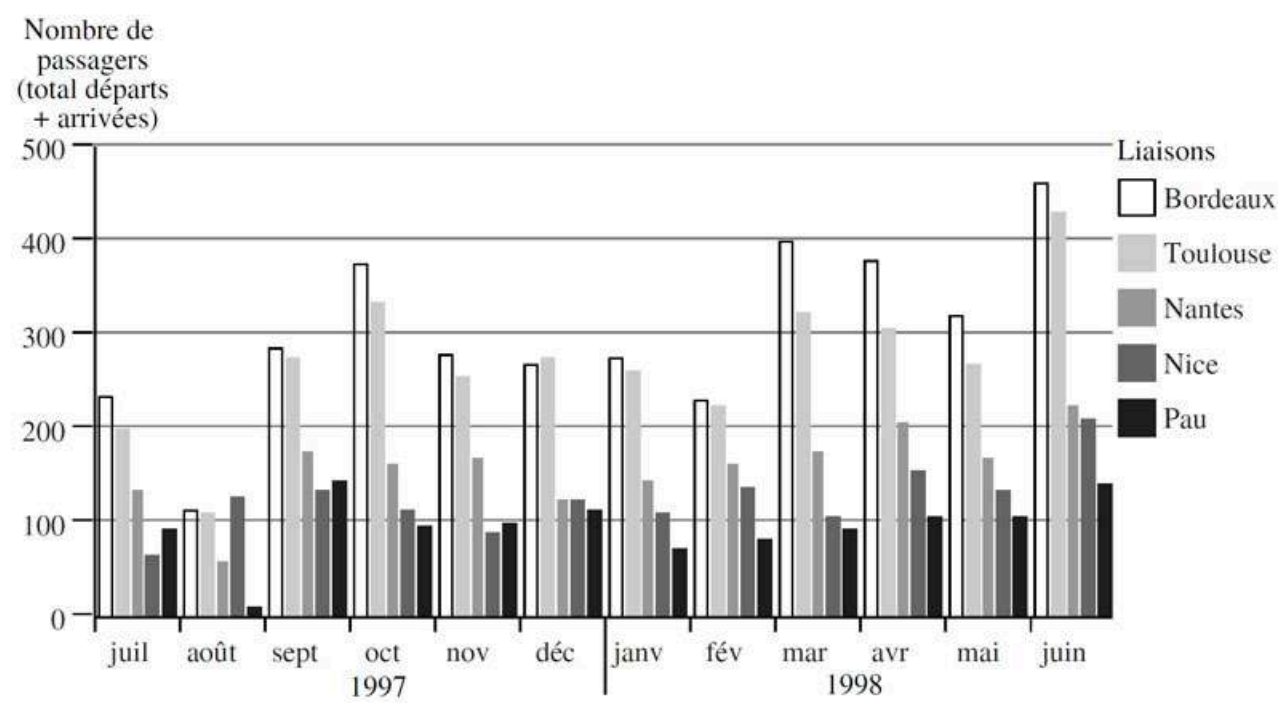

Source : Aéroport de Dijon-Bourgogne

La première remarque concerne la quasi absence de vols en août où Nice - destination touristique - fait cependant l'un de ses plus beaux scores, tandis que les quatre autres liaisons font le quart de leur trafic mensuel moyen. Bordeaux et Toulouse, villes d'affaires, se comportent en jumelles, montrant des pointes en juin, mars, avril et octobre. Nantes suit ce profil qui diffère un peu pour Pau dont la variation mensuelle de flux se calque plutôt sur celle de Nice, avec deux pointes en septembre et juin, (en avril, puis février et septembre pour Nice): Pau ville d'affaires ou ville de vacances? La proximité de Lourdes expliquerait-elle le peu de variation de flux concernant cette ligne, ou bien, la liaison aérienne très intéressante inciterait-elle les Bourguignons à partir par avion passer les fêtes de fin d'année à Pau comme à Nice : une enquête auprès des voyageurs nous éclairerait sans doute sur les raisons qui poussent chacun à prendre l'avion vers ces deux destinations.

En juillet 1997, avec l'arrivée en lice de Proteus Airlines à Dijon, trois destinations communes à Regional Airlines étaient annoncées : la concurrence était ouverte, la lutte allait-elle être pénalisante pour la société déjà installée ? (Griselin \& Ormaux, 1998).

La figure 10 fait état, pour ces trois liaisons communes, Bordeaux, Toulouse et Nantes, de résultats qui montrent tout à fait la complémentarité entre les deux hubs desservant Dijon. 
Fig. 10 : La concurrence entre Regional Airlines et Proteus Airlines : les flux aériens de Dijon vers les trois destinations communes aux deux compagnies

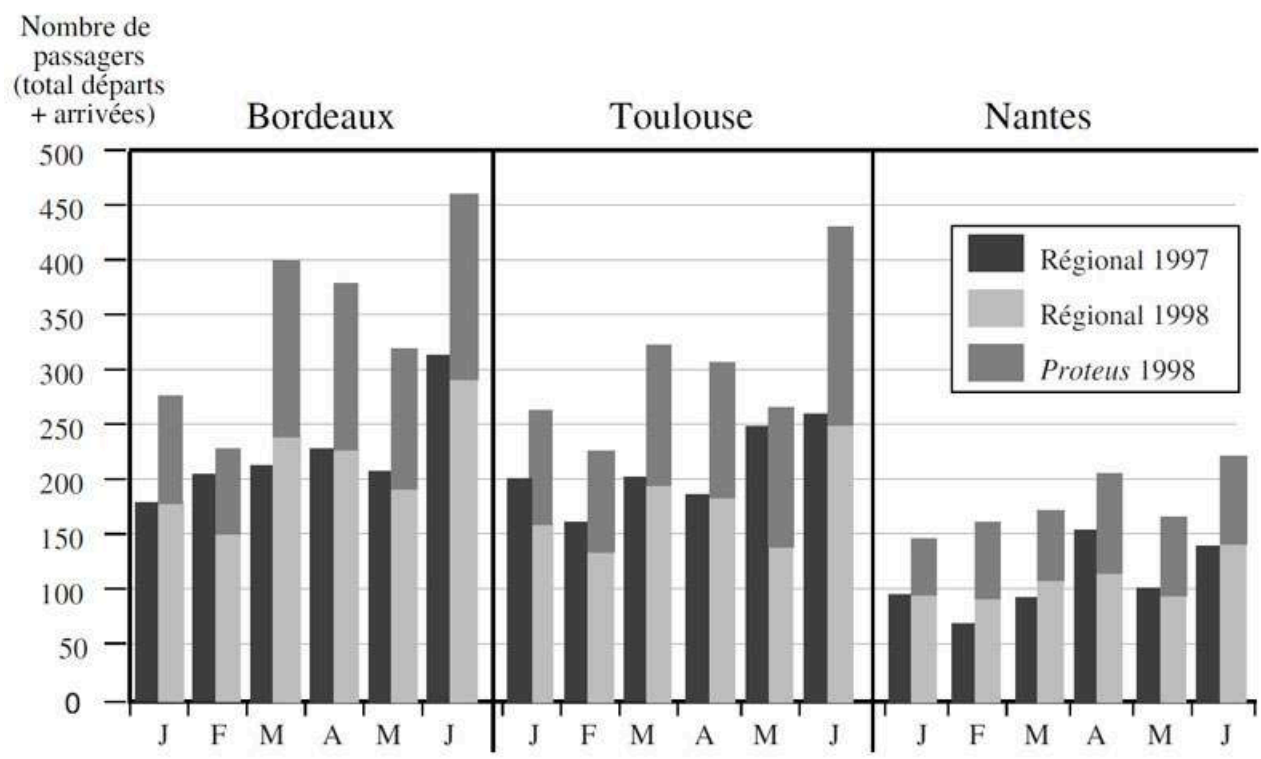

Source : Aéroport de Dijon-Bourgogne Regional Airlines opérait. Globalement, sur ces trois destinations communes, la compagnie centrée sur Clermont-Ferrand a maintenu son flux de passagers à $92 \%$ (3 281 en 1997 et 3022 en 1998), la perte allant de $0 \%$ du flux de 1997 sur Nantes à $15 \%$ sur Bordeaux. L'arrivée de Proteus se traduit par un accroissement de $51 \%$ du flux total de passagers (4 969 pour les six premiers mois de 1998 contre 3281 en 1997).

Proteus transporte actuellement $39 \%$ du flux passagers sur ces trois liaisons : sa part de marché est relativement constante de janvier à juin 1998, représentant globalement $26 \%$ du nombre de passagers total sur Bordeaux, 30 sur Toulouse et 19 sur Nantes.

incontestablement sur Toulouse que Proteus est le plus menaçant, et ce de façon mensuellement récurrente depuis janvier 1998. Nantes, avec une relative constance depuis six mois, reste le fief de Regional Airlines.

53 La concurrence est donc tout à fait loyale; à peu de chose près, l'entrée en lice de Proteus n'a fait que stimuler le trafic passager régulier à Dijon, la preuve que les deux hubs de Clermont et de Saint-Étienne offrent aux voyageurs des possibilités bien différentes et tout à fait complémentaires.

$\mathrm{Au}$ terme de ces analyses, il apparaît que le produit offert par les réseaux régionaux en étoile se situe dans un créneau intermédiaire entre le transport de masse tel que le $\mathrm{TGV}$, la route ou même l'avion long-courrier et la véritable aviation d'affaires supposant l'affrètement d'un appareil avec équipage. Les processus de filialisation et d'externalisation menés au sein des entreprises multiplient les nécessités de déplacements et de contacts que les moyens de communication électroniques ne sauraient remplacer. C'est donc toute une population, non plus seulement de PDG, mais de cadres et de commerciaux, qui a besoin de se déplacer rapidement et efficacement. Pour elle, et pour les territoires au sein desquels elle travaille, les réseaux en étoile qui se développent actuellement constituent véritablement une nouvelle donne. 
55 Alternative à une aviation d'affaires d'ailleurs peu répandue en France et perçue comme un luxe ostentatoire, les réseaux dont nous venons de parler sont également une chance de renouveau pour le transport aérien régional de passagers. En dehors de quelques lignes vedettes, celui-ci avait connu dans la décennie précédente un certain nombre de difficultés qui faisaient douter de son avenir, surtout dans un contexte de montée en régime de la grande vitesse ferroviaire. Or, même dans le cas envisagé ici d'un éloignement d'environ une heure par rapport à l'aéroport de raccordement, l'utilisation de tels réseaux s'avère intéressante, surtout dans la perspective d'un déplacement à la journée. De tels allers-retours sont tout à la fois rendus possibles et justifiés. La location de bureaux ou de salles de réunion à la journée dans les aéroports concernés peut encore améliorer l'instrument.

Quant aux villes sièges de l'aéroport-pivot, elles apparaissent comme de remarquables points de convergence au barycentre des lieux de départ et l'hypothèse qu'elles développent dans un avenir proche la fonction réunion, séminaire et congrès est à envisager. L'intense activité de discussion et d'échange qui règne à Clermont-Ferrand ou à Saint-Étienne en fin d'après-midi au moment où les passagers passent une demiheure en salle d'embarquement avant de repartir, est bien à l'image de cette fonction. Certaines des personnes présentes n'ont jamais posé et ne poseront peut-être jamais le pied dans la ville-pivot, mais pour toutes, celle-ci joue un rôle essentiel, non pas en tant que territoire mais comme pièce maîtresse d'un réseau. Dans ce contexte, être relié à un tel hub devient un enjeu et probablement un facteur actif de dynamique socioéconomique. La dimension réticulaire de l'espace s'affirme donc avec force, même comme ici à des niveaux très moyens de la hiérarchie urbaine.

Une réserve toutefois pour Dijon, la Bourgogne et la Franche-Comté : les deux réseaux en étoile, bien que se complétant mutuellement, n'offrent que très peu de possibilités de déplacements vers l'Est, c'est-à-dire vers le monde rhénan, l'Italie du Nord et l'ensemble de la dorsale européenne sur laquelle se localise une bonne part de la puissance et du dynamisme économique européen. Alors, il n'est pas étonnant qu'à l'aéroport de Dijon et à la Chambre de Commerce et d'Industrie de Bourgogne, on se prenne à rêver d'un réseau plus oriental et plus européen dont le hub serait à Dijon, ou au moins d'un réseau en étoile centré sur l'axe rhénan et auquel Dijon serait relié.

\section{BIBLIOGRAPHIE}

Boquet Y. (1988). - « Une nouvelle phase dans la restructuration du transport aérien aux ÉtatsUnis », L'Information Géographique, pp. 68-78.

Dacharry M. (1992). - « Transport aérien français et Marché Commun », Hommes et Terres du Nord, $\mathrm{n}^{\circ} 1$, pp. 34-39

Griselin M. et Ormaux S. (1997). - « Transports aériens en Franche-Comté », Images de FrancheComté, $\mathrm{n}^{\circ} 16$, pp. 21-24. 
Griselin M. et Ormaux S. (1998). - « La Franche-Comté et les réseaux aériens en étoile », Images de Franche-Comté, $\mathrm{n}^{\circ} 17$.

Mouhot P. (1990). - « Compagnies aériennes et réseaux aux Etats-Unis : évolution et typologie », Bulletin de l'Association des Géographes Français, n³, pp. 221-236.

Pavaux J. (1995). - Le transport aérien à l'Horizon 2000, ITA, 234 p.

Varlet J. (1997). - « La déréglementation du transport aérien et ses conséquences sur les réseaux et les aéroports ", Annales de Géographie, pp. 205-217.

\section{NOTES}

1. 18 destinations prises en compte ici, contre 17 au paragraphe précédent qui raisonnait sur les liaisons opérationnelle au 1er juillet 1998, excluant Toulon, abandonné à Dijon en décembre 1997.

\section{RÉSUMÉS}

Le développement des réseaux régionaux en étoile est-il en mesure de recomposer l'espace aérien au bénéfice de régions qui avaient longtemps souffert d'enclavement sur ce mode de transport? Si, par TGV et autoroute, la Franche-Comté est bien reliée à Paris, donc au trafic aérien long-courrier, elle était jusqu'alors très mal placée pour les liaisons aériennes avec le reste de la France ou le « proche Europe ». L'affirmation de Dijon à l'extrémité de branches de réseaux hub and spokes peut-elle modifier le rapport au déplacement aérien à moyenne distance, pour les habitants de Besançon et de l'Ouest comtois? Une analyse détaillée de la structure topologique des réseaux, une approche comparative des temps et des coûts entre l'avion, le train et la voiture et enfin une étude des flux sur 1997 et 1998 permettent d'apporter des éléments nuancés de réponse.

Is the development of a radial (hubs and spokes) systems of regional air transport capable of reorganising air space to the benefit of regions which have long suffered from isolation from this type of transport? Even if Franche-Comté is well linked to Paris by motorway and train (TGV), it remains badly placed for airlinks with the rest of France or adjacent European areas. Can the town of Dijon, now considered as a branch of the hub and spokes network, modify air mobility over moderate distances, especially for inhabitants in Besançon and West of the region? We present in this article a detailed analysis of the topological structure of the networks, a comparison of times and costs between aircraft, train and car, and a finally study of flows in 1997 and 1998.

Ist die Entwicklung regionaler Netze in Sternform geeignet, den Luftraum zugunsten der Regionen zu gestalten, die bei dieser Transportart lange unter Abschliessung gelitten haben? Wenn die Franche-Comté durch den TGV (Hochgeschwindigkeitszug) und die Autobahn gut mit Paris und dadurch mit dem Langstreckenflugverkehr verbunden ist, war sie bisher sehr schlecht bei den Luftverbindungen mit dem übrigen Frankreich und den näheren Teilen Europas versorgt. Kann die Zusicherung für Dijon, Teil des Netze « hub and spokes » zu werden, die Verbindungen für die Bewohner von Besançon und des Westens der Franche-Comté auf mittlere Entfernung 
modifizieren? Eine detaillierte Analyse der topologischen Struktur der Netze und eine vergleichende Betrachtung über Zeiten und Kosten zwischen Flugzeug, Bahn und Privatwagen sowie eine Studie über die Verkehrsströme 1997 und 1998 erlauben differenzierte Antworten auf diese Fragen.

INDEX

Mots-clés : Bourgogne, desserte, flux, Franche-Comté, hub and spokes, réseau en étoile, temporalité, transports aériens

Schlüsselwörter : Hub and spokes, Lufttransporte, sternförmiges Netz, Ströme

Keywords : air service, air transport, Burgundy, flows, Franche-Comté, hub and spokes, temporality

\section{AUTEURS}

\section{MADELEINE GRISELIN}

THÉMA CNRS - Université de Franche-Comté - 32, rue Mégevand 25030 Besançon Cedex SERGE ORMAUX

THÉMA CNRS - Université de Franche-Comté - 32, rue Mégevand 25030 Besançon Cedex 